\title{
Aceite de oliva: influencia y beneficios sobre algunas patologías
}

\author{
M. A. ZAMORA ARDOY, F. BÁÑEZ SÁNCHEZ1', C. BÁÑEZ SÁNCHEZ², \\ P. ALAMINOS GARCÍA ${ }^{3}$
}

Servicio de Farmacia Hospitalaria. Hospital "La Inmaculada". Huercal-Overa. Almería. ${ }^{1}$ Servicio de Medicina Interna. ${ }^{3}$ Servicio de Urgencias. Complejo Hospitalario de Jaén. ${ }^{2}$ Ingeniero Técnico Industrial.

\section{RESUMEN}

El olivo ha sido una de las bases de la agricultura de los países mediterráneos, con una gran importancia económica y social. El aceite derivado de su fruto, puede ser clasificado en diferentes tipos según su calidad, siendo el máximo exponente el denominado aceite virgen de oliva que aporta indudables beneficios para el mantenimiento de la salud, prevención de la enfermedad así como en una mejor evolución de esta cuando aparece. Existen estudios que demuestran estos beneficios en patologías como el cáncer sobre todo de pulmón y estómago (también de colon, endometrio y ovario). Patología gastrointestinal como úlcera péptica, litiasis biliar y sobre la motilidad gástrica. Sistema cardiovascular en patología coronaria, ictus e hipertensión arterial. Artritis reumatoide reduciendo el riesgo de su desarrollo y mejorando su evolución. Diabetes mellitus aumentando la sensibilidad a la insulina y reduciendo la presión arterial y nivel de lipoproteínas aterogénicas.

PALABRAS CLAVE: Aceite de oliva. Dieta mediterránea. Ácidos grasos monoinsaturados. Ácido oleico.

\begin{abstract}
The olive tree has been one of the agriculture bases in Mediterranean countries with a great economic and social significance. The oil derivative from it fruit can be clasified in diferents kinds acording with their quality, being the highest exponent the so-called pure olive oil that contribute in unquestionables benefits for the maintenance of health, illness prevention as well as a better evolution when the illness is present. The re are some studies that prove these benefits in pathologies like cancer specially breast and stomach cancer (colon, endometrium and ovary cancer too). Gastrointestinal pathology like peptic ulcer, cholelithiasis and gastric mobility. Rheumatoid arthritis decreasing it development risk and improving it evolution. Diabetes mellitus increasing insulin sensibility and decreasing blood pressure and atherogenic lipoprotein.
\end{abstract}

KEY WORDS: Olive oil. Mediterranean diet. Monounsaturated fatty acid. Oleic acid.

Zamora Ardoy MA, Báñez Sánchez F, Báñez Sánchez C, Alaminos García P. Aceite de oliva: influencia y beneficios sobre algunas patologías. An Med Interna (Madrid) 2004; 21: 138-142.

\section{INTRODUCCIÓN}

El olivo fue introducido en la Península Ibérica probablemente por los romanos y árabes y ha constituido la base de la agricultura de secano de los países de la cuenca mediterránea desde hace más de mil años junto a las rotaciones de cereales, leguminosas y al viñedo. Se trata en general de un sistema de producción basado en la excelente adaptación de la planta a las condiciones de sequía de su área de cultivo. Actualmente la superficie cultivada es de mas de 9 millones de hectáreas, con más de 700 millones de árboles, repartidos por diversas regiones del mundo, pero localizados en su mayor parte en la cuenca mediterránea. Según el Ministerio de Agricultura Pesca y Alimentación la superficie olivarera total española en el año 1992 fue de 2.141.100 hectáreas, lo que supone cerca del 10\% de la superficie agrícola nacional y el $24 \%$ de la superficie olivarera mundial; suponiendo la producción española un $30 \%$ de la pro- ducción mundial. Fundamentalmente el olivar cultivado se dedica a la producción de aceite y solamente 190.000 hectáreas a la producción de aceituna de mesa. Andalucía con el $60 \%$ de la superficie olivarera española produce el $80 \%$ del aceite de oliva, el resto se produce principalmente en Castilla-La Mancha, Extremadura y Cataluña $(1,2)$.

\section{CONCEPTOS GENERALES, DEFINICIÓN Y CUALIDADES DEL ACEITE DE OLIVA}

En 1986 el Convenio Internacional del Aceite de Oliva definió como "aceite de oliva" únicamente al aceite procedente del fruto del olivo, con exclusión de los obtenidos por disolventes, por procedimientos de reesterificación y de mezcla con aceites de otra naturaleza (3).

El aceite de oliva se diferencia de otros aceites vegetales en

Trabajo aceptado: 7 de octubre de 2003 